\title{
Preface for the Special Issue dedicated to Professor Julian E. Davies
}

\author{
The Journal of Antibiotics (2017) 70, 335-338; doi:10.1038/ja.2017.14
}

$T^{n}$ his special issue is dedicated to Dr Julian Edmund Davies, Professor Emeritus of Department of Microbiology \& Immunology at the University of British Columbia (UBC), Canada, in appreciation of his great contribution to research on antibiotics over the years (Figure 1).

It is the greatest pleasure for us, as the co-authors of a chapter entitled 'Aminoglycosides and Aminocyclitols' in the book 'Genetics and Biochemistry of Antibiotic Production' (edited by Leo C Vining and Colin Stuttard; Butterworth-Heinemann, Newton, MA, USA, 1995), to trace the footsteps of Julian in diverse research areas of antibiotics and microbial genetics.

The most recent proposal of Julian is the word 'parvome' ( parv $=$ small, - ome $=$ group) to describe the bioactive molecules in the microbial world, including antibiotics. This proposal has been made in September 2008, in a blog of the American Society for Microbiology, in order to obtain a better understanding of the true biological roles of natural products. This permits the application of rational approaches to a more effective exploitation of their discovery and use by mankind. In a recent review under the title of 'Introducing the parvome; bioactive compounds in the microbial world' (Davies J \& Ryan KS. Introducing the parvome; bioactive compounds in the microbial world. ACS Chem. Biol. 7, 252-259 (2012)), Julian and Katie Ryan described and discussed the existence and functions of the world of small bioactive organic molecules produced by living organisms.

Before proposing the term 'parvome', Julian together with his colleagues contributed significantly in a variety of research topics on antibiotics and microbial genetics, as exemplified by elucidations of the mechanism of streptomycin action in 1964 and that of

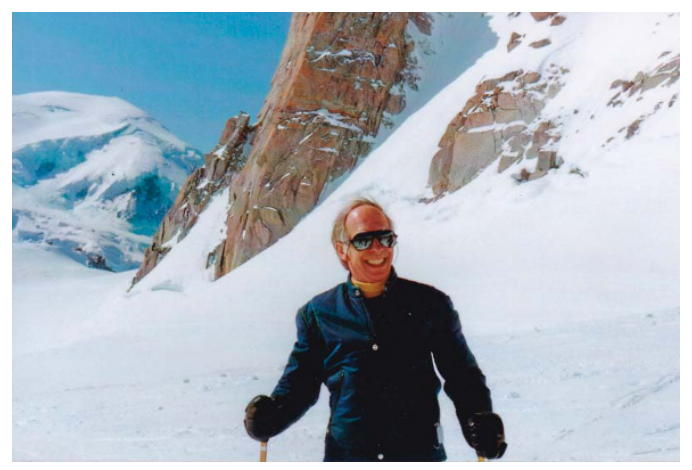

Figure 1 Recent photo of Professor Julian E. Davies. streptomycin resistance and numerous other aminoglycosides and other antibiotics in 1968.

One can trace Julian's footsteps, in more detail, in his autobiography published in 2003 (Davies J: Gathering no moss. Annu. Rev. Microbiol. 57, 1-27 (2003)). A description of his research subjects can be itemized as follows:

The inhibition of protein synthesis by streptomycin was elucidated, as its mechanism of action in 1964 at the Department of Bacteriology \& Immunology of the Harvard Medical School. The mechanism was further analyzed in detail, showing that streptomycin binds to a specific site on the $30 \mathrm{~S}$ ribosomal subunit, and causes mistranslation at low concentration. This topic has been extended through the aminoglycoside and aminocyclitol antibiotics, that is, spectinomycin, neomycin, and kasugamycin, by the brilliant structural studies of Venki.

Further elucidation of the mechanisms of aminoglycoside resistance in clinical important isolates and the inactivation of streptomycin by adenylylation in $R$-plasmid carrying bacteria was published in 1968. The adenylylating enzyme was found to also adenylylate spectinomycin. Inactivation of streptomycin by phosphorylation was confirmed in 1969 followed by the elucidations of enzymatic phosphorylation of neomycin and kanamycin by $R$-plasmid carrying resistant bacteria.

The enzymatic acetylation of neomycin, gentamicin Cla and kanamycin and related compounds at the 6'-amino group (AAC $\left(2^{\prime}\right)$ ) in 1974.

A companion discovery was published in 1973 showing that various species of Streptomyces producing aminoglycoside antibiotics were found to possess enzymes, which modified their antibiotics in a similar manner as found in clinically-isolated resistant bacteria. From these observations, it was suggested that the possibility of an evolutionary relations existed between the enzymes modifying aminoglycoside antibiotics in producing organisms and those inactivating these antibiotics in clinically resistant bacteria.

Later, the genes for aminoglycoside-modifying enzymes were subjected to cloning and expression in other host cells. The origin and evolution of antibiotic-resistance genes were analyzed and discussed in a review (Davies J. \& Davies D. Origins and evolution of antibiotic-resistance. Microbiol. Mol. Biol. Rev. 74, 417-433 (2010)).

A kanamycin-resistance gene of Escherichia coli carrying an $R$-factor was inserted into phage lambda, in 1975, and it was confirmed that the resistance determinant was a component of a transposable element, Tn5. Subsequently, the resistance determinant on Tn5 was successfully applied to constructing vectors for cloning of eukaryotic genes in the 
yeast, Saccharomyces cerevisiae. A tetracycline-resistance transposon Tn10 was studied similarly.

During subsequent gene cloning studies, restriction endonucleases of Providencia stuartii Pst1, Klebsiella pneumoniae Kpn1, Moraxella bovis Mbol and Bacillus circulans Bbu1 were discovered, isolated and purified.

Soon after the release of the NIH Guidelines on recombinant DNA, in June 1976, cloning of antibiotic-resistance genes was carried out extensively and the gene for aminoglycoside $2^{\prime \prime}$-nucleotidyltransferase $\left(\mathrm{ANT}\left(2^{\prime \prime}\right)\right)$ was cloned in 1977 and applied for drug monitoring of serum concentration of gentamicin.

Two plasmids of a Streptomyces fradiae strain were isolated in 1977 and their involvement in the biosynthesis of neomycin was suggested. Four different plasmids were isolated in an erythromycin-producing S. erythreus strain in 1982 and characterized by restriction endonuclease digestions.

Through expression of AAC $\left(6^{\prime}\right)$ of Streptomyces kanamyceticus in a strain of Streptomyces fradiae, which produces neomycin, increased resistance to and production of neomycin were observed.

Subsequently, a gene encoding an aminoglycoside phosphotransferase of Staphylococcus aureus, APH(3')-III, was cloned on a Streptomyces vector pIJ702, and introduced and expressed in a host Streptomyces lividans, in 1985. Since that time, a variety of interspecies expression studies have been conducted to enable the transfer and express genes. A simple and reliable method, which allows the direct transfer of shuttle plasmids between Streptomyces sp. and E. coli was established. In intergenic mating between $E$. coli and S. lividans, the Tra2 core of the IncP $\alpha$ plasmid RP4 was found to be required.

A gene for a new subfamily of pyridine nucleotide-disulfide oxidoreductase, coenzyme A disulfide reductase from $S$. aureus was cloned and overexpressed in E. coli to produce considerable amounts of enzyme protein for structural analysis and further studies on pathogenesis of $S$. aureus.

In domestic animals, in 2007, a highly fluoroquinolone-resistant and methicillin-resistant $S$. aureus was observed. It was consistent with a role for animal carriage in the dissemination of important human pathogens in the community.

Effects of subinhibitory concentrations of antibiotics on a variety of processes in $S$. aureus were investigated, by use of the promoter-lux gene fusion constructs. Some improved lux reporters for use in S. aureus were reported in 2009, and applicability of the promoter-lux reporters for drug discovery was proposed in 2010. By use of promoter-lux reporters, the effect of subinhibitory concentrations of cell wall-active antibiotics on virulence gene expression and biofilm formation in S.sureus was investigated in 2011.

In 2016, the construction of a high-throughput compatible gfp-luxbased multiplex promoter reporter platform to monitor $S$. aureus virulence genes expression in real time was reported. The application of this platform to identify natural products possessing activities modulating the expression of $S$. aureus virulence genes was also reported.

A genetic approach to studies of the pathogenesis of Mycobacterium was initiated in 1990 at the Institut Pasteur by use of a fast-growing species M.smegmatis. A method that allowed the stable integration of DNA fragment as a single site in the M.smegmatis chromosome was developed and interspecies gene transfer experiments were conducted.

In Vibrio cholerae, the Intl 1-mediated recombination of a geneVCR ( $V$. cholerae repeated sequence) cassette into a class 1 integron was investigated. A fosfomycin-resistant strain of Enterobacter cloacae was isolated, in 2001, from a water sample collected at a site near the mouth of a river in Canada.
In 1991, investigations of the pathogenicity of a filamentous fungus, genus Aspergillus, was initiated. The gene encoding for a ribotoxin, restrictocin, was cloned and characterized. In subsequent studies, ribotoxins were found to be a widespread group of proteins within the filamentous fungi.

The dynamics of DNA/RNA were studied in 1991 by elucidating the interaction of aminoglycoside antibiotics with DNA and inhibition of RNA self-splicing of group I introns was demonstrated. From the identification of superstructures, gene cassettes of chromosomal superintegrons were demonstrated to be the source of multiresistant integrons.

In 1993, at UBC, it was confirmed, by means of polymerase chain reaction (PCR) amplification of streptomycete $16 \mathrm{~S}$ ribosomal DNA sequence, that a number of antibiotic preparations for human and animal use were contaminated with chromosomal DNA of the antibiotic-producing organisms. Such DNA contained identifiable antibiotic-resistance gene sequences, thus, it was suggested that such contaminated DNAs might be the source of drug-resistance genes. Substantial bacterial DNA contamination was found, in 2004, in the animal feed-grade avoparcin, a glycopeptide antibiotic related to vancomycin. Nucleotide sequences related to the cluster vanHAX were present in the contaminated DNA, suggesting that the prolonged use of avoparcin as a feed-additive led to the uptake of glycopeptide-resistance genes by animal commensal bacteria.

Among 72 standard strains of E. coli in the ECOR Collection, 14 strains were found to show resistance to two or more antibiotics and by screening for the presence of integrons, four strains were found to carry a class 1 integron.

A survey of the heat shock response of streptomycin-producing strains was conducted, in 1991, and four heat shock proteins (HSPs) were elucidated. In 1994, at UBC, in a number of Streptomyces species, a variety of proteins were found to be phosphorylated on tyrosine residues, and the patterns of tyrosine phosphorylation were found to vary during the growth phase and under certain culture conditions. Thus, metabolic shifts during the growth cycle of Streptomyces, and possibly associated secondary metabolic pathways, were considered to be controlled by the action of protein kinases and phosphatases, as has been demonstrated in the signal transduction pathways in eukaryotic organisms. In 2002, it was proposed that an assay based on inhibition of aerial hyphae formation in Streptomyces might be applicable to the identification of protein kinase inhibitors. And in 2015, a method of screening for anticancer compounds by the use of Streptomyces kinase inhibitory assay was published and a number of active compounds were isolated by Dr Leng Chee Chang of the University of Hawaii.

Gene clusters involved in catalyzing the ring cleavage step and non-oxidative decarboxylation in the catabolism of aromatic compounds in Streptomyces strains were characterized in 1999, and a new project was initiated in 2004 to degrade polychlorinatedbiphenyl (PCB) by the use of Rhodococcus sp. There exist three catabolic plasmids encoding gene clusters for PCB degradation enzymes, and the presence of several oxygenase- and numerous dehydrogenase-coding genes was confirmed. The Rhodococcus RHAl strain possesses a large number of genes, potentially encoding multiple isozymes of the PCB-degradation pathway. Gene expression in the RHA1 strain was investigated in 2006, and the complete genome of the strain was sequenced.

Some streptomycetes are known to play important roles in biotransformation and biodegradation in nature. Studies on degradation of lignin-related aromatic compounds were conducted in 2011 by the use of Streptomyces sp. NL15-2K. 
In 2002, Julian proposed that the horizontal gene transfer (HGT), widely recognized in bacteria as the mechanism responsible for the widespread distribution of antibiotic-resistance gene, is also responsible for speciation and sub-speciation. And HGT mechanisms were also proposed to exist in eukaryotes. Two environmental strains of Delfinia spp. were found, in 2007, to carry class 3 integrons, which have seldom been reported only from pathogens, in which they are associated with antibiotic-resistance genes. Thus, the class 3 integron was considered to be horizontally transferred to Delfinia.

In 1995, at the UBC, the structure of U17, a thiol with low molecular mass, produced by Streptomyces clavuligerus was elucidated. Such a novel thiol substance shows a potential role as an antioxidant. Via an international collaboration, mycothiol was identified, in 1996, as a major thiol in number of actinomycetes, including Nocardia, Rhodococcus, Mycobacterium, Micromonospora, Actinomadula and Streptomyces spp.

In 2000, four novel natural products named the terragines $A, B, C$ and $\mathrm{D}$ were obtained from soil libraries in a Streptomyces lividans host following genetic implementation of the host. The term 'combinatorial biosynthetic libraries' was proposed.

In 2010, Julian described that the actinobacteria, including Actinomycetales and all related genera are the richest source of small molecule diversity on the planet. These compounds have an incredible variety of chemical structures and biological activities. Also, in 2011, Julian described under the title of 'Streptomycetes are special: arcane applications' that one cannot escape the fact that streptomycetes are an inexhaustible source of bioactive small molecules and that their therapeutic uses are boundless.

In 2001, screening for natural products in his laboratory was expanded into lichens, which are symbiotic associations between fungi, green algae and/or cyanobacteria. Some molecular genetic techniques using PCR, genomic library construction and heterologous expression made an approach possible to screen for novel chemical entities in lichens. An undescribed Streptomyces obtained from the surface of a lichen produced a new enediene antibiotic, uncialamycin, and the structure and in vitro activity against Gram-positive and Gramnegative human pathogenic bacteria of uncialamycin were studied together with Raymond Andersen. Also, from a culture of a Streptomyces strain isolated from the lichen, four new congeners of undecose nucleoside herbicidin were isolated in 2014.

In 2014, Julian proposed, from the observation of anti-virulence activities of the human gut metabolome, that microbes can communicate through the production and sensing of small molecules. Within the complex ecosystem formed by commensal microbes living in and on the human body, it is likely that these molecular messages

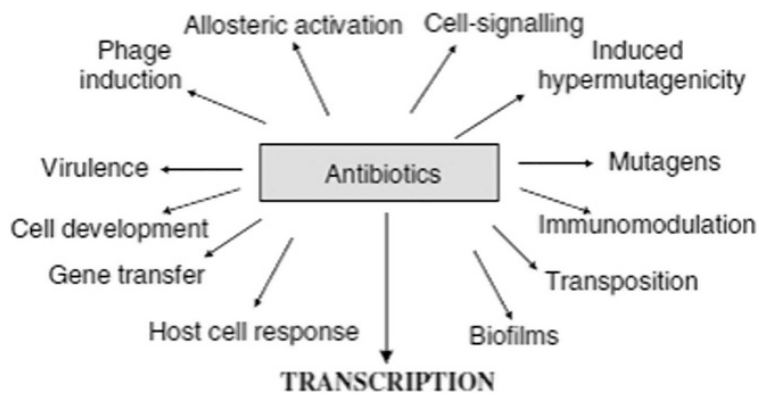

Figure 2 Antibiotics as signalling molecules. A full color version of this figure is available at The Journal of Antibiotics journal online. are used extensively during the interactions between different microbial species as well as with host cells.

In 2002, transcriptional modulations of bacterial expression by subinhibitory concentration of antibiotics were described. From these studies, it was suggested that the detection of pharmaceutically useful natural product inhibitors could be effectively achieved by measuring transcription activation at low concentration in high-throughput assays using appropriate bacterial promoter-reporter constructs.

The wide range of effects of subinhibitory antibiotic concentrations were summarized in a review entitled 'The world of subinhibitory antibiotic concentrations' (Davies J, Spiegelman GB \& Yim G. The world of subinhibitory antibiotic concentrations. Curr. Opin. Microbiol. 9, 445-453 (2006)).

In 2006, Julian published significant arguments on the diverse bioactive natural antibiotics in the reviews entitled 'The truth about antibiotics' (Yim G, Wang HH \& Davies J. The truth about antibiotics. Int. J. Med. Microbiol. 296, 163-170 (2006)) and 'Are antibiotics naturally antibiotics?' (Davies J. Are antibiotics naturally antibiotics? Ind. Microbiol. Biotechnol. 33, 496-499 (2006)). Antibiotics were also defined, in 2007, as signalling molecules based on their primary effects in the maintenance of microbial communities in the environment (Yim G, Wang HH \& Davies J. Antibiotics as signalling molecules. Phil. Trans. R Soc. B 362, 1195-1200 (2007)) (Figure 2).

Small molecules produced by microbes were found to have many different functions in nature, and they also interact with proteins and RNA with high specificity and have been shown to be regulators and effectors of diverse biochemical reactions.

In 2011, Julian wrote in a review under the title of 'How to discover new antibiotics: harvesting the parvome', concluding that combined with advanced chemical separation and characterization techniques, the construction of large chemically diverse libraries of bioactive compounds for the therapeutic applications from natural products is a realistic near-term goal.

In 2016, he suggested the possibility that low molecular weight effectors were gradually replaced by polypeptides as polymerizing reactions became more complex, but retained some ability to interact with original receptor sites.

Julian gave an alarm, in 2013, that natural products became important industrial products and, consequently, the absence of any knowledge of the roles and functions of small molecules in nature was discussed. It is necessary to redirect enquiry into the natural roles of those molecules and the ways in which they regulate microbial populations.

Also, in 2013, Julian gave concluding remarks in a review entitled 'Antibiotic resistance in and from nature' (Davies J. Antibiotic resistance in and from nature. Microbiol. Spectrum 1, 0005-2012 (2013)) that: (1) Are antibiotics really antibiotics as defined by their use as anti-infective agents? (2) Are antibiotics produced and released naturally in the environment? At present, there is no evidence for the presence of antibiotics in the soils. (3) It has been shown definitively that natural resistomes are common and exist in different environmental niches, and (4) most resistance enzymes possess binding sites for antibiotics. Might there be alternative biological functions yet to be recognized? Finally, Julian concluded that it is interesting to consider how the development and use of antibiotics would have been different if we had approached their use from a One Health perspective, and that we perhaps this would have avoided the problem of antibiotic resistance.

Before closing this preface, we should appreciate Julian's contribution to the Journal of Antibiotics serving as an editorial board member during 1971 and 2006, and as an emeritus member thereafter. In 1983, Julian worked on full amendment and completion of the 'Instruction 
to Authors' of the journal, and in 1991, worked on the contemporary definition of the term 'antibiotics'.

Guest Editors for the Special Issue Dedicated to Professor Julian E Davies.

\section{CONFLICT OF INTEREST}

The authors declare no conflict of interest.
Kunimoto Hotta ${ }^{1}$ and Morimasa Yagisawa ${ }^{2}$ ${ }^{1}$ Function Water Foundation, Shinagawa-ku, Tokyo, Japan and ${ }^{2}$ Department of Drug Development and Regulatory Science, Faculty of Pharmacy, Keio University, Minato-ku,

Tokyo, Japan

E-mail: khotta@fwf.or.jp oryagisawa2013@gmail.com 